\title{
NTRK3 Fusion Positive
}

National Cancer Institute

\section{Source}

National Cancer Institute. NTRK3 Fusion Positive. NCI Thesaurus. Code C132236.

Indicates that the expression of a NTRK3 fusion has been detected in a sample. 